\title{
Modelling the joint choice of activity timing and duration
}

\author{
Dick Ettema $^{\mathrm{a}, *}$, Fabian Bastin ${ }^{\mathrm{b}}$, John Polak ${ }^{\mathrm{c}}$, Olu Ashiru ${ }^{\mathrm{c}}$

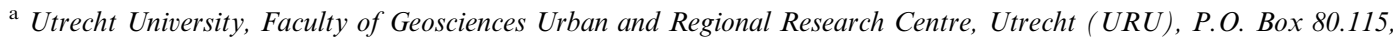 \\ 3508 TC Utrecht, The Netherlands \\ ${ }^{\mathrm{b}}$ CERFACS, 42, Avenue Gaspard Coriolis, 31057 Toulouse Cedex 01, France \\ ${ }^{\mathrm{c}}$ Centre for Transport Studies, Department of Civil and Environmental Engineering, Imperial College London, \\ Exhibition Road, London SW7 2AZ, United Kingdom
}

Received 18 January 2006; received in revised form 17 February 2007; accepted 29 March 2007

\begin{abstract}
This paper develops a model of activity and trip scheduling that combines three elements that have to date mostly been investigated in isolation: the duration of activities, the time-of-day preference for activity participation and the effect of schedule delays on the valuation of activities. The model is an error component discrete choice model, describing individuals' choice between alternative workday activity patterns. The utility function is formulated in a flexible way, applying a bell-shaped component to represent time-of-day preferences for activities. The model was tested using a 2001 data set from the Netherlands. The estimation results suggest that time-of-day preferences and schedule delays associated with the work activity are the most important factors influencing the scheduling of the work tour. Error components included in the model suggest that there is considerable unobserved heterogeneity with respect to mode preferences and schedule delay. (C) 2007 Elsevier Ltd. All rights reserved.
\end{abstract}

Keywords: Activity based modelling; Time allocation; Activity and trip timing

\section{Introduction}

The recent growth of interest in activity-based methods has focused particular attention on travellers' decision-making process regarding the timing and duration of their participation in activities. Models of timing and duration choice have direct application to a wide range of demand management policies and are at the core of many recent activity based modelling systems. However, to date in the literature these two dimensions of activity participation have been largely treated separately, despite the compelling observation that in general, the benefit that an individual derives from participating in an activity will depend inter alia both upon the time at which the activity is undertaken and the amount of time devoted to the activity. Moreover, since many of the influences on the timing and duration of activity participation (such as the perceived quality of available travel modes and activity opportunities and the intensity with which an individual undertakes activities) will

\footnotetext{
* Corresponding author.

E-mail address: d.ettema@geog.uu.nl (D. Ettema).
} 
vary by individual and context and some are inherently difficult to completely characterise via conventional travel or time use data, it is likely that such decisions will be characterised by a significant degree of heterogeneity.

This paper proposes a model for the simultaneous choice of the timing and duration of activities and travel mode. The model is based on earlier work by the authors (Ashiru et al., 2004; Ettema et al., 2004), which formulated the model of activity timing and duration as a discrete choice system. It extends the previous work in two important aspects. First, it accommodates the influence of explicit schedule constraints, in the form of a schedule delay concept, thus providing an important point of connection between the recent activity scheduling literature and the earlier literature on trip re-scheduling. Second, it accounts for both observed and unobserved heterogeneity in activity scheduling behaviour. Observed heterogeneity is accommodated through explicit segmentation of model parameters according to socio-demographic characteristics whereas residual, unobserved inter-personal heterogeneity is accommodated through allowing key parameters to vary randomly across individuals.

The structure of the paper is as follows. Section 2 of the paper provides a brief review of the existing literature on activity timing and duration choice. Section 3 introduces the theoretical approach, which assumes that the marginal utility derived from activities encompasses two distinct components; one derived from the duration of activity involvement and the other derived from activity participation at a particular time-of-day, possibly in relation to existing anchor points, such as the work start time. Section 4 discusses the data that were used to test the model empirically. Section 5 describes the estimation methodology. Particular attention is given to the use of advanced optimisation techniques needed to estimate the non-linear utility function expressing individuals' timing and duration preferences. Section 6 discusses the estimation results and the conclusions that can be drawn with respect to individuals' decision making regarding timing and duration of activities. The paper closes with some overall conclusions and a discussion of future research directions.

\section{Relevant literature}

According to activity based travel theory (Ettema and Timmermans, 1997), trips can be regarded as a necessary means to connect spatially remote activities that will logically precede or follow these activities. This implies that the timing of trips not only depends on trip characteristics that vary by time-of-day (such as travel time and delays) but also on preferences with respect to the timing and duration of activities. Consequently, when modelling trip-timing decisions, these should be regarded in the context of the activity-scheduling process (e.g. Ettema and Timmermans, 2003).

With respect to modelling the timing and duration of activities, various approaches have been taken within the activity-based framework. Focusing on timing decisions, a first group of models (Bowman and Ben-Akiva, 1998; Arentze and Timmermans, 2005), although applying widely different decision-making mechanisms, have essentially treated the timing of activities as being a choice between a limited number of discrete time intervals. For instance, Bowman and Ben-Akiva (1998) conceptualise the timing of activities as the choice between the morning, afternoon or evening. Models of this type are based on the assumption that for particular activities, certain periods of the day are more attractive, resulting in higher utilities. A limitation of this approach is that only a limited number of broad periods are assumed, and that the exact timing of activities is not directly modelled. In addition, it is assumed that utility is gained from the execution of activities as a whole, thereby ignoring the variation in the utility gained during the execution. This may be a suitable approach for shorter activities, but in case of activities with a long duration, such as work, it is desirable to allow for variation in the attractiveness of the activity during its execution. This is especially the case if one is interested in for instance responses of travellers to congestion which involve retiming of the commute trip, resulting in retiming of the work activity.

To account for variations in the duration of activities, several authors (e.g. Ettema et al., 1995; Bhat, 1996) have proposed the use of hazard based duration models. Hazard models treat duration as a continuous variable and describe the probability of exiting an activity conditional on the time already spent in the activity. The hazard rate is typically affected by a series of socio-economic variables. A drawback of hazard modelling, however, is that it provides only a statistical approach to modelling distributions of durations, which lacks an underlying behavioural theory. In response to this shortcoming, various approaches have described the sched- 
uling of activities in continuous time, using a utility based framework. The central idea of these approaches is that for each time-of-day $t$, there exists a marginal utility (which may vary over time), expressing the utility gained from one time unit of activity participation. The idea of a time of day specific marginal utility (or utility rate) was first introduced in the context of activity scheduling by Polak and Jones (1994), building on the work of Winston (1982). The idea was refined further by Ettema and Timmermans (2003), who proposed specific functional forms for the utility rate functions.

Although the Polak-Jones-Ettema-Timmermans (henceforth, PJET) models provide a flexible approach to modelling activity-timing decisions; two problems need to be addressed. The first problem is the neglect of the duration component within their marginal utility formulations. Many activities are likely to be subject to fatigue or satiation effects, implying that the utility derived from one time unit of activity participation diminishes with increasing duration. The basic PJET models in contrast, assume that one unit of activity engagement at time-of-day $t$ will always yield the same utility, irrespective of the duration of activity engagement.

A way to account for satiation effects is offered by time allocation models, which are based on the seminal work of Becker (1965), who treated time as a finite resource, which can be allocated to activities, resulting in a certain level of utility. Time allocation is in this view regarded as an optimisation problem under the restriction of a fixed amount of resources (time). The duration of activities is then determined by the allocation of time to activities such that the overall utility is maximised. Becker's model was elaborated by, amongst others, Evans (1971) and DeSerpa (1971) in order to account for the consumption of goods given consumption rates, prices and the available monetary budget. Other extensions of this approach include the modelling of time allocation on the household level (Zhang et al., 2002) and the specification and testing of advanced time-dependent utility functions (Joh et al., 2003). If the utility derived from an activity is defined as a log-function of the time spent on an activity, the time allocation model can be formulated as a system to be estimated using for instance seemingly unrelated regressions (Kitamura, 1984; Bhat and Misra, 1999). An important property of this type of formulation is that the marginal utility of activities decreases with their duration, representing the onset of activity fatigue. Although the Becker-type models are able to describe how individuals maximise utility by allocating time to activities, they do not take into account the preferences that individuals have with respect to the timing of activities. Recently, Ashiru et al. (2004) and Ettema et al. (2004) have formulated and empirically tested models that combine the time-of-day dependent marginal utility function of the PJET-models and the duration dependent Becker-type models.

A second problem of the JPET formulation is the assumption that the timing of activities is purely based on continuous marginal utility functions associated with activities. This assumption overlooks the fact that the timing of many activities is partially determined by constraints such as work or school arrangements and opening hours of stores and facilities (Hägerstrand, 1970). Such constraints may lead to discontinuities in the marginal utility function relative to anchor points such as work start time or the opening and closing time of facilities.

An approach explicitly accounting for such discontinuities is the schedule delay approach (Small, 1982). This approach focuses especially on the desired start time of activities. It is typically assumed that associated with each activity there is a preferred start time. Likewise, the trip to this activity has some preferred arrival time (PAT). Deviations from the preferred arrival time (schedule delays) result in a negative utility. In Small's model trip utility is not only a function of travel time $t$, but also of schedule delay:

$$
V_{t}=\alpha t+\gamma_{1} \mathrm{SDE}+\gamma_{2} \mathrm{SDL}
$$

where SDE is an early schedule delay, defined as $\max \left(\left(\mathrm{PAT}-t^{\mathrm{a}}\right), 0\right)$; SDL is a late schedule delay, defined as $\max \left(\left(t^{\mathrm{a}}-\mathrm{PAT}\right), 0\right)$; and $t^{\mathrm{a}}$ is the actual arrival time.

This formulation implies that there is overlap between the schedule delay approach and the JPET models. In particular, the SDL-parameter can be interpreted as the (constant) marginal utility of the activity to start after arrival, since each time unit the activity starts later results in a loss of utility of $\gamma_{2}$. Likewise, the SDEparameter can be interpreted as being associated with the duration of the activity preceding the trip in a similar way, since arriving too early goes at the cost of the activity at the trip origin. On the other hand, there are also differences between the approaches. For instance, the schedule delay approach does not explicitly include any valuation of utility of activity participation. In addition, the valuation of an activity that starts before the preferred arrival time is not directly addressed. 
From a conceptual point of view, one can argue that the JPET models assume that activities can be scheduled at any time, independent of timing constraints. This scheduling process is then based on some intrinsic time-of-day dependent utility. In reality, however, the timing of most activities is at least to some extent guided by constraints, even if applying to other activities. For instance, the timing of an activity such as "taking a walk" which can principally take place any time, will be determined by obligations to spend time on work and household obligations, limiting the available time window. In empirical studies, it may therefore be difficult to properly disentangle the pure time-of-day preference and the impact of constraints when estimating the marginal utility functions. The schedule delay approach, on the other hand, is much more focused on the direct effect of scheduling constraints on the timing of activities and trips.

Schedule delay models have mostly been applied to model trip departure time choice in the context of single activities, such as work. Occasionally, the schedule delay approach has been applied to model the choice between activity patterns, combining schedule delay of the work trip with duration effects of work and other activities (Hess et al., 2007). A limitation, however, is that the schedule delay approach assumes the existence of some anchor point representing the optimal point in time to arrive or start an activity. This may work well for activities such as work, which are relatively fixed in time, but is more cumbersome for e.g. leisure activities.

From the above, one can conclude that three sources may be identified that influence the timing and duration of activities and thereby the emergence of activity patterns:

- 'intrinsic' time-of-day preferences, represented by continuous marginal utility functions (Ettema, 2005; Polak and Jones, 1994);

- satiation effects, stemming from diminishing marginal returns, as described in the Becker-type time allocation models (Kitamura, 1984; Bhat and Misra, 1999);

- scheduling constraints of the Hägerstrand type as described by the schedule delay approach (Small, 1982).

In most modelling studies to date, these sources of scheduling utility have been treated in isolation. An exception is Ettema et al. (2004), who combine time-of-day and duration dependent utility in one framework. Another notable exception is Vovsha and Bradley (2004), who also combine duration and time-ofday dependent utility in their model. In particular, they assume that the utility of a tour consists of utility components related to the start time of the tour, the duration of the tour and the arrival time home of the tour. In each of these components, utility is described as a linear function of clock time or duration, by using continuous shift variables, in which socio-demographic characteristics interact with time. Timing constraints are explicitly dealt with by excluding certain time intervals from the set of feasible times. In terms of the above classification of scheduling utility components, the tour duration is related to the satiation effect, whereas the tour departure and arrival time preferences may be conceptually linked to both intrinsic time of day preferences and scheduling constraints. A particularly strong point of the Vovsha and Bradley approach is that it can be estimated in a straightforward way using conventional activity-trip diaries. The framework proposed in this paper is related to the Vovsha and Bradley's approach in that it assumes that the utility of a daily work tour consists of various utility components based on duration and time-of-day. However, our approach is based on a different specification of utility components, which is in line with the three sources of utility (time-of-day, satiation, scheduling constraints) outlined before. This implies the following characteristics:

1. Instead of utility being a linear function of duration, we account for a satiation effect, by using a marginal utility function which decreases with duration.

2. To represent intrinsic time-of-day preferences we use a highly flexible, non-linear utility specification.

3. We explicitly include interactions between activities in the duration dependent utility component to represent trade-offs in time allocation between different parts of the day.

Thus, the framework outlined in this paper extends the models recently developed by Ashiru et al. (2004) and Ettema et al. (2004), including both time-of-day dependent components and duration dependent components, with a schedule delay component. The resulting model is tested in the context of the combined choice of work tour and mode under a road-pricing regime. In specifying and testing the model, particular attention is 
given to incorporating both observed and unobserved heterogeneity, by including socio-demographic factors and error components.

\section{Theoretical framework}

\subsection{Theoretical model}

Our theoretical model follows some basic assumptions put forward by a number of other authors, namely that:

1. Individuals derive a certain utility from allocating time to activities (Becker, 1965; Yamamoto and Kitamura, 1999) and this utility depends both on the amount of time allocated and the time of day at which participation in the activity takes place (Ettema et al., 2004).

2. Individuals derive a certain (dis)utility from the time spent travelling (Ben-Akiva and Lerman, 1985).

3. Individuals aim at optimising the utility of their overall activity pattern, being the sum of the individual activity and trip utilities (Becker, 1965; Jara-Diaz, 1998a,b; Meloni et al., 2004).

Mathematically speaking, we assume that individuals maximise their utility by solving:

$$
\max V=\max \left(V^{\mathrm{T}}+V^{\mathrm{A}}\right)
$$

where $V^{\mathrm{T}}$ is the total utility derived from trips and $V^{\mathrm{A}}$ the total utility derived from activity participation. This specification is in line with Vovsha and Bradley in that the overall utility of a tour or utility pattern is composed of activity and trip utilities. These utilities are the sums of the utilities of individual trips and activities:

$$
\begin{aligned}
V^{\mathrm{T}} & =\sum_{m} V_{m}^{\mathrm{T}} \\
V^{\mathrm{A}} & =\sum_{n} V_{n}^{\mathrm{A}}
\end{aligned}
$$

Since our study focuses on timing and duration effects associated with activities, the utility of each individual trip $m$ is defined as a relatively simple function of travel time $\left(R_{m}\left(s_{m}\right)\right)$ and travel cost $\left(C_{m}\left(s_{m}\right)\right)$ associated with trip $T$ made at start time $s_{m}$. In addition, a constant $D_{m}^{l}$ is included to represent the constant utility of a trip made by mode $l$ :

$$
V_{m}^{\mathrm{T}}\left(s_{m}\right)=D_{m}^{l}+v R_{m}\left(s_{m}\right)+\mu C_{m}\left(s_{m}\right)
$$

where $\mu$ and $v$ are the travel time and cost parameter, respectively. It is noted that additional trip characteristics can be added without materially changing the approach. Scheduling costs, which represent the disutility of the diversion of some preferred arrival time for the trip, are not included in the utility of trips. Instead, these are represented in the utilities of activities through the implications for activity duration and timing. It is also noted that socio-demographics are included as adjustments to a specific parameter as follows:

$$
\beta^{p}=\left[\beta_{0}+\sum_{j=1}^{J} \beta_{j} \delta_{j}\right]
$$

where $\beta^{\mathrm{p}}$ is the parameter for a person with socio-demographics defined by a $J$-dimensional vector $p$, containing dummy-variables; $\beta_{0}$ is the base value of the parameter; $\beta_{j}$ is the adjustment for the $j$ th dummy variable; and $\delta_{j}$ takes value 1 if the $j$ th element of $p$ is unity and zero otherwise. The utility derived from an activity depends, as noted before, on three distinct elements:

- the time-of-day at which an activity is performed. In this respect we assume that there is some intrinsic preference for the time-of-day at which certain activities are undertaken;

- the duration of the activity, assuming that with increasing duration fatigue effects will come into play, resulting in a diminishing utility with increasing duration; 
- the start time of the activity, relative to some reference point. In this respect, we assume that specific constraints, such as work hours and store-opening hours, directly affect the timing of activities.

With respect to timing and duration, the above implies for instance that the first minute spent on an activity may be valued differently than the 10th or 50th minute, but the 10th minute may be valued differently when engaged in at 7.00 AM or 2.00 PM. In addition, the start time of the activity will have an effect on the utility, which is, however, independent of the duration of the activity. To capture these effects, we define the utility $V_{n}^{\mathrm{A}}$, derived from engagement in activity $n$ as:

$$
V_{n}^{\mathrm{A}}=f\left(V_{n}^{\mathrm{H}}\left(t_{n}, s_{n}\right), V_{n}^{\mathrm{D}}\left(t_{n}\right), V_{n}^{\mathrm{S}}\left(s_{n}, s_{n}^{*}\right)\right)
$$

where $V_{n}^{\mathrm{H}}$ is the time-of-day dependent utility, depending on start time $s_{n}$ and duration $t_{n} ; V_{n}^{\mathrm{D}}$ is the duration component, depending on duration $t_{n} ; V_{n}^{\mathrm{S}}$ is the schedule delay component depending on the preferred activity start time and the actual start time $s_{n}$.

Focusing first on the three separate components, the time-of-day component is specified as the baseline utility profile, specifying the user benefit of being involved in an activity at a particular time of day. The time-ofday dependent utility is best understood in terms of the marginal utility $V_{n}^{\prime \mathrm{H}}(s)$ specifying the amount of utility gained from participation during one time unit at time of day $s$. The assumption underlying our framework is that individuals have a preferred time-of-day for engagement in an activity, at which time the marginal utility is maximal. Engagement before of after this time then leads to a gradually lower marginal utility. A bell shaped marginal utility function meets these specifications. Ettema et al. (2004) tested various bell shaped functions empirically and found a symmetrical Cauchy distribution to be an efficient function to describe time-of-day dependent utility:

$$
V_{n}^{\prime \mathrm{H}}(s)=\frac{1}{c_{n} \pi\left[\left(\frac{s-b_{n}}{c_{n}}\right)^{2}+1\right]} * V_{\max , n}
$$

In this function, $b_{n}$ defines the optimum location, that is where the utility is a maximum, $c_{n}$ defines the width of the curve (which is symmetrical), which gives the time period in which an acceptable level of utility is gained, and finally $V_{\max , n}$ scales the Cauchy distribution (see Ettema et al., 2004 for examples of the effects of the parameters on the utility shape). The essence of the marginal utility component $V_{n}^{\prime \mathrm{H}}$ is to express that the utility derived from activity engagement intrinsically depends on the time-of-day. In this case we have assumed a bell-shaped curve to represent the time-of-day dependent utility, implying that the high marginal utility is concentrated in one period. Without materially changing the approach, however, alternative functions may be specified, implying for instance multiple periods with a high marginal utility. Socio-demographic variables can principally affect the time-of-day dependent utility through $V_{\max , n}, b_{n}$ or $c_{n}$ in the same way as indicated in Eq. (6).

Given the marginal utility function $V_{n}^{\prime \mathrm{H}}(t)$, the utility gained from activity $n$ can be determined by integration if the start time $s_{n}$ and duration $t_{n}$ are known:

$$
V_{n}^{\mathrm{H}}\left(s_{n}, t_{n}\right)=\frac{V_{\text {max }, n}}{\pi}\left(\arctan \left(\frac{s_{n}+t_{n}-b_{n}}{c_{n}}\right)-\arctan \left(\frac{s_{n}-b_{n}}{c_{n}}\right)\right)
$$

With respect to the duration dependent utility $V_{n}^{\mathrm{D}}$, we assume that the utility follows a logarithmic function, as proposed for example by Yamamoto et al. (2000) and Bhat and Misra (1999):

$$
V_{n}^{\mathrm{D}}\left(t_{n}\right)=\eta_{n} \ln \left(t_{n}\right)
$$

This results in the following marginal utility:

$$
V_{n}^{\prime \mathrm{D}}\left(t_{n}\right)=\frac{\eta_{n}}{t_{n}}
$$

An important implication of this function is that marginal utility decreases with increasing duration, representing a satiation effect, which is intuitively plausible. Socio-demographics can affect the duration dependent utility by modifying the constant $\eta$, as specified in Eq. (6). It is noted that Eq. (11) in combination with the 
additive formulation of Eq. (4) implies that the utilities of different activities are independent of each other. That is to say, the utility derived from $n$ minutes of participation in activity $A$ is independent of the time spent in activity $B$. There are, however, instances in which time spent on one activity is valued higher if more time is spent on another activity. For instance, time spent on recreation activities may give a higher utility if more time is spent on working on a given day. To allow for such interactions, we extend the utility function (11) to:

$$
V_{n}^{\mathrm{D}}\left(t_{n}, t_{m}\right)=\eta_{n} \ln \left(t_{n}\right)+\eta_{n m} \ln \left(t_{n}\right) \ln \left(t_{m}\right)
$$

In this formulation $\eta_{n m}$ represents a parameter indicating the degree to which two activities complement or substitute each other. A positive parameter would indicate that two activities complement each other, whereas a negative sign would point at substitution between activities.

Finally, we define the schedule delay dependent utility of an activity $n$ as:

$$
V_{n}^{\mathrm{S}}=\gamma_{n}^{e} \mathrm{SDE}_{n}+\gamma_{n}^{l} \mathrm{SDL}_{n}
$$

where SDE and SDL are the early and late schedule delay, respectively, defined as (see Hess et al., 2007):

$$
\begin{aligned}
& \operatorname{SDE}_{n}=\max \left(0,\left(s_{n}^{*}-s_{n}\right)\right) \\
& \operatorname{SDL}_{n}=\max \left(0,\left(s_{n}-s_{n}^{*}\right)\right)
\end{aligned}
$$

where $s_{n}^{*}$ is the preferred start time of the activity. Having specified the components $V_{n}^{\mathrm{D}}, V_{n}^{\mathrm{H}}$ and $V_{n}^{\mathrm{S}}$, the total utility derived from an activity, $V_{n}^{\mathrm{A}}$, is specified as a function of the respective parts. It is recognised that the components can be combined in different functional specifications (additive, multiplicative, logarithmic or combinations of these). Since this paper constitutes a first exploration of these utility components, we have chosen to use the most straightforward additive function:

$$
V_{n}^{\mathrm{A}}\left(t_{n}, s_{n}, s_{n}^{*}\right)=V_{n}^{\mathrm{H}}\left(t_{n}, s_{n}\right)+V_{n}^{\mathrm{D}}\left(t_{n}\right)+V_{n}^{\mathrm{S}}\left(s_{n}, s_{n}^{*}\right)
$$

Because the components $V_{n}^{\mathrm{D}}$ and $V_{n}^{\mathrm{H}}$ are scaled by $\eta_{n}$ and $V_{\max , n}$, respectively, it is not necessary to add weights to each component. The utility of activity $n$ is thus defined by:

$$
V_{n}^{\mathrm{A}}\left(s_{n}, t_{n}, s_{n}^{*}\right)=\left[\frac{1}{\pi}\left(\arctan \left(\frac{s_{n}+t_{n}-b_{n}}{c_{n}}\right)-\arctan \left(\frac{s_{n}-b_{n}}{c_{n}}\right)\right)\right] V_{\max , n}+\eta_{n} \ln \left(t_{n}\right)+\gamma_{n}^{e} \mathrm{SDE}_{n}+\gamma_{n}^{l} \mathrm{SDL}_{n}
$$

\subsection{Operational model}

The operational model is applied to a home-based work tour and is defined in the current study as follows. Following the approach of Polak and Jones (1994), we assume that travellers choose the departure time of trips from home to work and from work back to the home. This effectively divides the day into three periods (pre-work, work, and after-work), which we regard as single activities of which the utility is defined by Eq. (16). It is recognised that regarding the daily activity schedule effectively as consisting of three activities is a strong simplification, which is likely to have an effect on the outcomes. In particular, regarding the pre-work and after-work period as single composite activities disregards the differences that exist between the individual activities included in these categories in terms of timing and duration preferences. This implies that the various utility functions derived for the pre-work and after-work period represent some average that may not hold for any of the individual activities, suggesting that much heterogeneity is associated with differences between activities will be missed. Nevertheless, we believe that the approach suffices to illustrate the interplay between timing, duration and schedule delay considerations, especially in the scheduling of daily work arrangements. Deriving utility specifications for a wider spectrum of activities is regarded as an important objective for future work in this area. The above implies that the total utility of a commuters' activity pattern $i$ can be formulated as:

$$
V_{i}=V_{1}^{\mathrm{T}}+V_{2}^{\mathrm{T}}+V_{1}^{\mathrm{A}}+V_{2}^{\mathrm{A}}+V_{3}^{\mathrm{A}}+\varepsilon_{i}
$$


with $V_{n}^{\mathrm{T}}$ as defined in Eq. (5) and $V_{n}^{\mathrm{A}}$ as defined in Eq. (16). In this respect, early and late schedule delays are defined relative to the current work start time or start time of the after work activity, which is assumed to be the preferred start time. It is recognised that the current work start time is not necessarily the preferred start time. However, the fact that the variations in work start time in the stated preference survey were large (up to $2 \mathrm{~h}$ ) makes this assumption defendable. In addition, formulations were tested allowing for interactions between the durations of activities (see Eq. (12)), such that the total utility gained from activity duration is expressed as:

$$
\begin{aligned}
V^{\mathrm{D}}\left(t_{\text {prework }}, t_{\text {work }}, t_{\text {afterwork }}\right)= & \eta_{\text {pre-work }} \ln \left(t_{\text {pre-work }}\right)+\eta_{\text {work }} \ln \left(t_{\text {work }}\right)+\eta_{\text {after-work }} \ln \left(t_{\text {after-work }}\right) \\
& +\eta_{\text {prework*work }} \ln \left(t_{\text {pre-work }}\right) \ln \left(t_{\text {work }}\right)+\eta_{\text {pre*afterwork }} \ln \left(t_{\text {pre }}\right) \ln \left(t_{\text {afterwork }}\right) \\
& +\eta_{\text {work*afterwork }} \ln \left(t_{\text {afterwork }}\right) \ln \left(t_{\text {work }}\right)
\end{aligned}
$$

In the current study we will assume that an individual chooses between a limited number (say $N$ ) of feasible activity patterns $\left[P_{1}, \ldots, P_{N}\right]$ characterised by total utilities $\left[V_{1}, \ldots, V_{N}\right]$. It is assumed that the choices made in the SP experiment reflect the preferences for certain time allocation patterns. In particular, the chosen alternative may be considered to be the closest match to an individual's unconstrained allocation outcome. Therefore, the discrete choice data can be used to disentangle the marginal utility functions that guide time allocation on a continuous scale. Assuming a Gumbel distribution for $\varepsilon_{i}$ in Eq. (17) leads to a multinomial logit model as the base specification.

Thus, it is assumed that discrete choice theory provides an adequate framework to model the choice of activity patterns. As noted previously, our model can account for heterogeneity associated with socio-demographic characteristics. However, heterogeneity in preferences may also arise from unobservable sources, such as taste variations. Especially when various types of utilities are combined which may interact and partly overlap, as in this study, it is expected that significant variation between individuals will exist with respect to their valuation of various timing and duration aspects and their interactions. Accounting for such variations is deemed crucial in this study. Such variations can be accounted for by more flexible formulations of the traditional discrete choice models (Train, 2003; Hensher and Greene, 2003) in which error components are included in the utility function. In particular, in this study, we assume that the evaluation of a certain utility component may vary from person to person, leading to addition of a random parameter, following a normal distribution with zero mean and unknown standard deviation. A complication in this case is that we work with stated preference data, in which multiple observations per person are available. In order to take account of correlations between choices that are expressed by the same individual, we assume here that the error component terms only vary from individual to individual. Taking the schedule delay formulation as an example this is expressed as:

$$
U_{n, i r}^{\mathrm{S}}=\left(\gamma_{n}^{e}+\zeta_{n, i}^{e}\right) \mathrm{SDE}_{n}+\left(\gamma_{n}^{l}+\zeta_{n, i}^{l}\right) \mathrm{SDL}_{n}
$$

where $U_{n, i r}^{\mathrm{S}}$ is the schedule delay utility of activity $n$ experienced by individual $i$ in replication $r$. In this formulation, $\zeta_{n, i}^{e}$ is an error component with mean zero and some standard deviation $\sigma_{n, i}^{e}$ to estimate. In the current study, error components will mainly be used to test for the heterogeneity in the evaluation of attributes. That is to say error components can in principle be added to any parameter $b_{n}, c_{n}, V_{\max , n}, \eta_{n}, \gamma_{n}^{e}$ or $\gamma_{n}^{l}$ in Eq. (16). We will in addition use an error component term associated to sets of modes, in order to take a possible nest structure into account.

\section{Stated preference data}

The model proposed in Section 2 was empirically tested using a stated preference data set, collected on various sites in The Netherlands in 2001 as part of a project to assess commuters' potential responses to various road user-charging schemes. Respondents were recruited by means of detailed screening and quota control criteria in which drivers undertaking work, employers business, shopping and social and leisure tours were selected.

The stated preference experiments involved respondents being offered realistic choices between alternative tour patterns. In order to avoid highly unattractive or highly unrealistic SP alternatives, these alternatives were 
developed based on the characteristics of the individual's current tour, which could include any type of activity. For the present study, however, only work tours were used.

During the SP experiment respondents were provided with (a) re-timing options involving shifts earlier or later relative to the most temporally constrained activity (work); (b) activity duration options; (c) total twoway travel time options; and (d) total road price charge options. In the survey, a public transport tour, similar to the most attractive existing PT tour, was offered as an alternative for the road pricing options. Thus, each respondent was offered four basic alternatives:

1. A car tour with departure times around the current departure times.

2. A car tour with departure times earlier than the current departure times.

3. A car tour with departure times later than the current departure times.

4. A public transport tour.

Each alternative was presented in terms of:

1. Departure time of the commute trip.

2. Duration of the outbound and return commute trip.

3. Work start time and work duration.

4. Departure time of return trip.

5. Duration of return trip.

Each respondent was presented eight choice sets.

The data set provides data regarding the relevant choice dimensions incorporated in the model: activity timing and duration, trip duration and mode choice and is therefore suitable to test the model. To define the prework and post-work period in terms of their duration, it was decided to end the after-work period and start the pre-work at midnight. The underlying motivation is that it in this way the after-work activity includes the evening activities, while the pre-work activity includes sleeping and the activities before work, such as personal care and breakfast. To evaluate the model, data for respondents who indicated that their current tour was a work trip were selected, as the resulting home-based tour is considered most likely to represent a daily activity pattern. After tests for data consistency and completeness, this resulted in some 1,382 observed choices, delivered by 188 individuals. For each subject, a limited number of socio-demographics were available, along with information regarding their working arrangements.

Stated preference data were used to test the modelling framework since it has some important advantages over revealed preference data for our purpose. Most importantly, it allows us to estimate utility specifications based on trade-offs between different alternative activity schedules in which factors such as timing, duration and schedule delay are varied systematically. Using revealed preference data would provide us with detailed observed activity patterns, but not with alternative activity patterns that are needed to derive the model parameters in a discrete choice framework. It is recognised that the current stated preference data implies a serious simplification in that daily activity schedules are reduced to three activity types. However, since our objective is to demonstrate the principles of our approach, we feel that the use of these data are appropriate. Further work is needed to develop stated preference procedures that allow for estimating utility components for much more detailed activity classes. More advanced stated preference approaches could include a wider range of activity classes, varying numbers of activities and different activity sequences. Based on such data, more specific utility functions can be estimated, which can be applied to real life settings with greater validity than the current demonstration. As for any model including the concept of schedule delays based on preferred arrival times, such an application would require that data are collected regarding arrival preferences in such a way that it can be generalised to the population as a whole.

\section{Estimation procedure}

Estimating the model outlined previously involves finding the parameters that maximise the goodness-of-fit of the logit model. Following Ben-Akiva and Lerman (1985) the log-likelihood function is formulated as: 


$$
L L(\theta)=\frac{1}{I} \sum_{i=1}^{I} \sum_{n} y_{n}^{i} \log P_{n}^{i}
$$

where $P_{n}^{i}$ is the probability that individual $i$ chooses activity pattern $n ; y_{n}^{i}$ is a dummy variable indicating whether individual $i$ chooses alternative $n$; and $I$ is the population size.

The parameters $\theta$ are then computed by solving:

$$
\max _{\theta} \phi(\theta)=L L(\theta)
$$

The highly non-convex character of the log-likelihood function (20), caused by the non-linear utility specification, leads us to consider nonlinear programming approaches, especially trust-region methods. The main idea of a trust-region algorithm involves the calculation, at iteration $k$ (with current estimate $\theta_{k}$ ), of a trial point $\theta_{k}+s_{k}$ by approximately maximising a model $m_{k}$ of the objective function inside a trust region defined as

$$
B_{k}=\left\{\theta \text { such that }\left\|\theta-\theta_{k}\right\| \leqslant \Delta_{k}\right\},
$$

where $\Delta_{k}$ is called the trust-region radius. We can for instance use a quadratic model:

$$
m_{k}(s)=L L\left(\theta_{k}\right)+s^{\mathrm{T}} \nabla_{\theta} L L\left(\theta_{k}\right)+\frac{1}{2} s^{\mathrm{T}} H_{k} s,
$$

where $H_{k}$ is a symmetric approximation of the Hessian $\nabla_{\theta \theta}^{2} L L\left(\theta_{k}\right)$. The predicted and actual increases in the value of the objective function are then compared by computing the ratio:

$$
\rho_{k}=\frac{L L\left(\theta_{k}+s_{k}\right)-L L\left(\theta_{k}\right)}{m\left(\theta_{k}+s_{k}\right)-m\left(\theta_{k}\right)} .
$$

If this ratio is greater than a certain threshold, set to 0.01 in our tests, the trial point becomes the new iterate, and the trust-region radius is (possibly) enlarged. More precisely, if $\rho_{k}$ is greater than 0.75 , we set the trustregion to be the maximum between $\Delta_{k}$ and $2 s_{k}$, otherwise we set $\Delta_{k}=0.5 \Delta_{k}$. If the ratio is below the bound, the trial point is rejected and the trust region is shrunk by a factor of 2 , in order to improve the correspondence of the model with the true objective function. We have followed Conn et al. (2000) in our choice of the parameters.

We additionally constrain the parameters $V_{\max , n}$ end $c_{n}$ of the marginal utility (8) to be strictly positive, since its integral (10) is discontinuous at $c_{n}$ equal to zero. Moreover we assume that $V_{n}^{\mathrm{H}}$ is positive, so $V_{\max , n}$ and $c_{n}$ must be of the same sign. Note, however, that if $V_{\max , n}$ or $c_{n}$ converges to zero, both corresponding time of day marginal utility and its integral vanish. Therefore, if some of the non-negativity constraints are active at the solution, the associated time of components do not add useful information to the model, and can be excluded from it. The resulting model is then unconstrained, and can be estimated using standard nonlinear programming techniques.

In order to estimate the error component parameters, we have used 1024 randomised Sobol draws (Owen, 1998) per individual for each error component. Experimentations with increasing numbers of draws suggest that stable results are obtained with this number of draws.

\section{Empirical results}

Using the above algorithm, the model according to Eqs. (16) and (17) was estimated. While this slightly differs from the specification given in Eq. (17), alternative specific constants were added to the early and late car trip alternatives, as well as to the public transport alternative, since this significantly increased the goodness-of-fit of the models. It is recognised that adding alternative specific constants for time-of-day is undesirable in a model of activity timing and scheduling, which is based on a continuous time representation. In the non-error component logit models, adding alternative specific constants added very significantly to the goodness-of-fit of the models. In the error component specifications, however, it is found that the time-of-day constants are no longer significant. This suggests that the error component logit models provide a better base for developing models of activity timing and duration. In addition, schedule delays are included only for the work 
activity in the final specifications. In the estimation process we tested specifications with schedule delays applying to the after-work activity but we did not find significant effects of these. A potential reason is that the afterwork activity basically includes a wide variety of activities, some of which are tied to specific constraints while others can freely be scheduled. The effect of schedule delays may therefore differ widely between individual settings.

Based on an initial MNL formulation various models were estimated (Table 1). Model 1 is a base formulation, which only includes alternative specific constants and duration dependent utility components. Model 2 is an extended model, including also schedule delay and time-of-day dependent components. Model 3 is a model specification, which also includes error components for schedule delay parameters and the public transport constant. Model 4, finally, includes interactions between the duration-based utilities of different activities as well as error components. Various alternative specifications of Eq. (19) were tested, allowing for different interactions. The specification with all three interactions was found to perform best and is presented here. From the goodness-of-fit measures it is concluded that including time-of-day and schedule delay components results in a significant improvement as also indicated by a likelihood ratio test (Table 2). This finding suggests that when

Table 1

Estimation results of models of activity pattern choice

\begin{tabular}{|c|c|c|c|c|}
\hline & Model 1 & Model 2 & Model 3 & Model 4 \\
\hline $\begin{array}{l}\text { Alternative specific constants } \\
D^{\text {car,early }} \\
D^{\text {car,late }} \\
D^{\text {public transport }}\end{array}$ & $\begin{array}{l}-1.08^{* * *} \\
-2.44^{* * *} \\
-2.03^{* * *}\end{array}$ & $\begin{array}{l}-0.46^{* * *} \\
-0.74^{* * *} \\
-2.25^{* * *}\end{array}$ & $\begin{array}{l}0.20 \\
-0.43 \\
-5.51^{* * *}\end{array}$ & $\begin{array}{l}0.15 \\
-0.45 \\
-5.59^{* * *}\end{array}$ \\
\hline $\begin{array}{l}\text { Duration dependence utility parameters } \\
\eta_{\text {pre-work }} \\
\eta_{\text {work }} \\
\eta_{\text {post-work }}\end{array}$ & $\begin{array}{l}1.99^{* *} \\
1.26^{* *} \\
0.81\end{array}$ & $\begin{array}{l}6.70^{* * *} \\
0.58 \\
-0.37\end{array}$ & $\begin{array}{l}6.79^{*} \\
-0.03 \\
-1.69\end{array}$ & \\
\hline $\begin{array}{l}\eta_{\text {pre-work } * \text { work }} \\
\eta_{\text {pre-work*post-work }} \\
\eta_{\text {work } * \text { post-work }} \\
\eta_{\text {post-work,male }} \\
\eta_{\text {pre-work,high-educ }}\end{array}$ & $\begin{array}{l}0.46 \\
1.04^{*}\end{array}$ & $\begin{array}{l}1.40^{* *} \\
3.80^{* *}\end{array}$ & $\begin{array}{l}2.59^{* * *} \\
0.40\end{array}$ & $\begin{array}{l}0.85^{* *} \\
0.61 \\
-0.86^{* *} \\
2.63^{* *} \\
2.43\end{array}$ \\
\hline $\begin{array}{l}\text { Schedule delay parameters } \\
\gamma_{\text {work }}^{\mathrm{e}} \text { (early schedule delay) } \\
\gamma_{\text {work }}^{1} \text { (late schedule delay) }\end{array}$ & & $\begin{array}{l}-0.0141^{* * *} \\
-0.0093^{* *}\end{array}$ & $\begin{array}{l}-0.067^{* * *} \\
-0.031^{\text {*** }}\end{array}$ & $\begin{array}{l}-0.064^{* * *} \\
-0.032^{* * *}\end{array}$ \\
\hline $\begin{array}{l}\text { Time-of-day dependent utility parameters } \\
b_{\text {work }} \text { (time-of-day with highest utility) } \\
c_{\text {work }} \text { (width of Cauchy curve) } \\
V_{\text {max,work }} \text { (maximum utility at time } b_{\text {work }} \text { ) } \\
V_{\text {max,work,high-educ }}\end{array}$ & & $\begin{array}{l}569.05^{* * *} \\
69.696^{* * *} \\
6.24^{* * *} \\
1.18\end{array}$ & $\begin{array}{l}580.93^{* * *} \\
83.72^{* * *} \\
14.47^{* * *} \\
-1.95\end{array}$ & $\begin{array}{l}573.28^{* * *} \\
78.29^{* * *} \\
16.32^{* * *} \\
-3.24\end{array}$ \\
\hline $\begin{array}{l}\text { Travel time parameters } \\
v_{\text {car }} \\
v_{\mathrm{PT}}\end{array}$ & $\begin{array}{l}-0.0076^{* * *} \\
-0.0061^{* *}\end{array}$ & $\begin{array}{l}-0.0005 \\
-0.0003\end{array}$ & $\begin{array}{l}-0.0092^{*} \\
-0.0123\end{array}$ & $\begin{array}{l}-0.0072^{*} \\
-0.0090\end{array}$ \\
\hline $\begin{array}{l}\text { Travelcost parameters } \\
\mu_{\mathrm{car}} \\
\mu_{\mathrm{PT}}\end{array}$ & $\begin{array}{l}-0.040^{* * *} \\
0.0002\end{array}$ & $\begin{array}{l}-0.075^{* * *} \\
0.0050\end{array}$ & $\begin{array}{l}-0.180^{* *} \\
0.0108\end{array}$ & $\begin{array}{l}-0.180^{* *} \\
0.0086\end{array}$ \\
\hline $\begin{array}{l}\text { Error terms } \\
\zeta_{\mathrm{PT}} \\
\zeta_{\mathrm{SDE} \text {,work }} \\
\zeta_{\mathrm{SDL} \text {,work }}\end{array}$ & & & $\begin{array}{l}4.028^{* * *} \\
0.042^{* * *} \\
0.030^{* * *}\end{array}$ & $\begin{array}{l}3.984^{* * *} \\
0.042^{* * *} \\
0.032^{* * *}\end{array}$ \\
\hline $\begin{array}{l}L L(0) \\
L L(\beta)\end{array}$ & $\begin{array}{l}-1904.77 \\
-1323.16\end{array}$ & $\begin{array}{l}-1904.77 \\
-1219.09\end{array}$ & $\begin{array}{l}-1904.77 \\
-952.286\end{array}$ & $\begin{array}{l}-1904.77 \\
-948.346\end{array}$ \\
\hline
\end{tabular}

${ }^{*}$ Significant at $\alpha=0.10$.

*** Significant at $\alpha=0.05$

*** Significant at $\alpha=0.01$. 
Table 2

Likelihood ratio tests of nested models

\begin{tabular}{lll}
\hline & Model 2/model 1 & Model 3/model 2 \\
\hline$\chi^{2}$ of likelihood ratio & 208.14 & 533.61 \\
Degrees of freedom & 6 & 3 \\
Significance level & $<0.01$ & $<0.01$ \\
\hline
\end{tabular}

modelling activity-scheduling processes, all three factors (duration, time-of-day and schedule delays) should be taken into account in order to obtain a realistic behavioural description and adequate sensitivity to policy scenarios. The error component models (models 3 and 4) in turn constitute a very significant improvement in model fit (Table 2). Partly, this suggests that taste variations exist between travellers with respect to their timing and duration preferences. In addition, the error component found for the public transport constant suggests a violation of the IIA property of the MNL formulation. On the other hand, it is recognised that the data used to test our framework has limitations with respect to the explanatory variables that can be included in the model. For instance, treating the time before and after work as one single activity is a simplification that ignores the wide variety in the way individuals spend their leisure time, which has different impacts on their scheduling behaviour of the work activity. Thus, the highly significant error components also capture these unobserved variations in activity scheduling conditions. Although the modelling framework used in this study suffices for demonstrating the importance of combining various timing and duration components, the error components suggest that more elaborate models are needed to describe the activity scheduling process in more detail. The estimation results are discussed in the remainder of this section with particular focus on the error component models (models 3 and 4). In terms of goodness-of-fit, model 4 outperforms model 3, suggesting that the interactions explain more of the observed behaviour than the main effects of duration only. However, since the models are not nested, we cannot test the significance of this improvement.

The alternative specific constants $\left(D^{\text {car,early }}, D^{\text {car, late }}, D^{\text {public transport }}\right)$ suggest that travellers have a lower preference for a public transport tour than for car options. Models 1 and 2 suggests that they value an earlier or later retiming of their current tour negatively, but this effect is not significant in the error component specification.

With respect to the utility of activity durations, the results of models 2 and 3 suggest that activity involvement leads to an increased utility only for the pre-work activity ( $\eta_{\text {pre-work }}$ ) (although only marginally significant in the error component logit models). Engagement in post-work activity ( $\eta_{\text {post-work,male }}$ ) is valued positively by males. Highly educated individuals gain a higher utility from the pre-work activity $\left(\eta_{\text {pre-work,high-educ }}\right)$, but this effect is not significant in the error component model. We do not find a significant effect of the duration of the work activity on utility, probably because the utility of work comes through the time-of-day dependent utility component. Interestingly, the model in which interactions between the duration components are included gives significant parameter estimates for the interaction between pre-work and work (positive) and work and after-work (negative). This suggests that time spent before work and during work are complementary, but that work and the time after work are substitutes. In other words, individuals who work longer attach a higher utility to the pre-work activity (and reverse), whereas they attach a lower utility to the after-work activity (and reverse). This finding stresses the importance of allowing for such inter-activity interactions in activity scheduling models.

The parameters describing the time-of-dependent utility of work $\left(b_{\text {work }}, c_{\text {work }}, V_{\text {max,work }}\right)$ are highly significant and consistent in models 2 to 4 . The parameters suggest that the highest marginal utility of work is derived at $573 \mathrm{~min}(9.33 \mathrm{AM})$ and is lower before and after that time. The shape of the marginal utility curve is displayed in Fig. 1. It is noted that the time-of-day dependent utility implicitly represents a duration dependency, since a longer engagement in work will result in a higher utility. This may account for the non-significance of the work duration parameter $\eta_{\text {work }}$ in models 2 to 4 .

As indicated by the SDE and SDL parameters, both early and late schedule delay are valued negatively, with late schedule delay, surprisingly, being less negative as early schedule delay. These findings are in contrast with the literature in this area (e.g. Small, 1982; De Jong et al., 2003). It should be noted, however, that when arriving before $b_{\text {work }}(9.33 \mathrm{AM}$ ) late schedule delay also leads to an increasing loss in time-of-day dependent utility, which can possibly explain the discrepancy. In addition, it is noted that the effects of early and late schedule delay on the after work activity were tested empirically. One would expect that such delays are rel- 


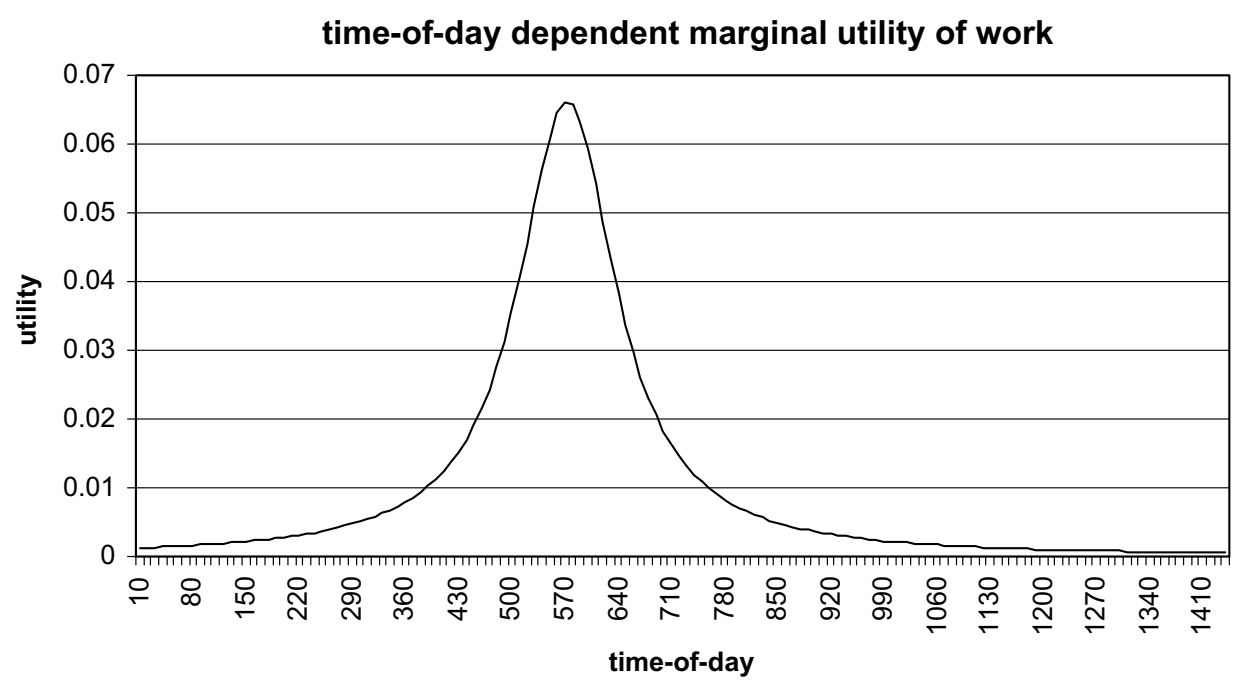

Fig. 1. Time-of-day dependent marginal utility curve.

evant for activities such as collecting children at a day care centre or household obligations. However, these effects were not found to be significant, possibly due to the fact that the after work activity is a composite of a wide variety of possible activities. Further research should address potential differences between these activities with respect to timing and duration preferences.

With respect to travel time parameters $\left(v_{\mathrm{car}}, v_{\mathrm{PT}}\right)$ and travel cost parameters $\left(\mu_{\mathrm{car}}, \mu_{\mathrm{PT}}\right)$ we find, as expected, significant negative parameters for car. The parameters for travel time and travel cost of public transport are, however, not significant. Apparently, the constant disutility of public transport ( $\left.D^{\text {public transport }}\right)$ is more important than the variable aspects like travel time and costs.

The various time-of-day, duration and schedule delay based utility components together suggest that there are rather subtle relationships between the various components of the utility of activities and trips, which may partly overlap and correlate. Based on the estimation results we can conclude, however, that scheduling constraints pertaining to the work activity, the time-of-day dependent utility of work, the duration of the pre-work time and the duration of the post-work time (for males) seem to be the most important determinants in deciding how to schedule the work tour. In addition, evidence is found for interactions between activities with respect to the utility derived from activity duration. Travel time seems to be important to the extent that it affects the involvement in work in the period between 8:30 and 10:30 when the marginal utility of work is highest.

As noted previously, various error components were tested, maintaining only those that are significant. The final models include error components for $D^{\text {public transport }}, \mathrm{SDE}_{\mathrm{work}}$ and $\mathrm{SDL}_{\mathrm{work}}$, suggesting a high degree of unobserved heterogeneity with respect to the associated parameters and preferences. A first conclusion is that including the error components has a large effect on the goodness-of-fit (Table 2), suggesting that the activity scheduling process that we are modelling is surrounded by much unobserved heterogeneity. It should be noted, however, that the experiment we have used is a simplified representation that does not necessarily include all relevant variables that influence activity scheduling and timing decision in reality. More elaborate experiments should make clear to what extent the unobserved heterogeneity in this study includes the effect of omitted variables. For the mode error component $\zeta_{\mathrm{PT}}$ (associated with the public transport dummy), the existence of heterogeneity is in line with findings in other studies indicating that travellers may have intrinsic preferences for a particular mode. In addition, the mode error component seems to affect the alternative specific constants. The public transport constant is larger (negative), while the early and late rescheduling constants are not significant in this model. The error components associated with early and late schedule delay, $\zeta_{\text {SDE, work }}$ and $\zeta_{\text {SDL,work }}$, suggest that significant variation exists in the valuation of early schedule delay. Such variation may be due to differences in the household situation, such as obligations for childcare and serve passenger trips, differences in commute distances or differences in the flexibility of work hours. This taste variation may also affect the early and late rescheduling constants. It is also noted that other parameter are affected 
by the inclusion of the error components. The valuation of pre-work time ( $\left.\eta_{\text {pre-work }}\right)$ in general, but also by highly educated travellers ( $\left.\eta_{\text {pre-work,high-educ }}\right)$, suggests that this parameter may capture part of heterogeneity, but the classification into education strata is not in itself relevant. Finally, the scaling of travel time and cost parameters seems to be affected by inclusion of the error components.

\section{Conclusions}

This paper has proposed a utility-theoretic framework for timing and duration preferences embedded in a multi-dimensional choice model, which can be formulated in a flexible way as an error component logit model. Doing so, the proposed framework combines a utility-theoretic underpinning with a very flexible formulation of time and duration preferences, which includes the effect of activity duration, the effect of time-of-day of activity involvement and the effect of start time of the activity relative to some anchor point. It is noted that from a theoretical point of view, the time-of-day dependent utility and the valuation of schedule delay overlap to some extent. It may therefore be difficult to disentangle these effects in empirical studies. The model framework further includes socio-demographic and context variables, that may affect individuals' valuation of timing and duration.

The model was tested empirically using a Dutch stated preference data set, accounting for context variables such as gender and education level. Various model specifications were estimated, differing with respect to the timing and duration related variables that are included. Some important conclusions can be drawn from the estimated models. First, the three distinct aspects of utility (duration, time-of-day and schedule delay) all appear to have some effect on activity scheduling preferences. It should be noted, however, that the aspects may overlap and may therefore be hard to disentangle. The effects differ between activities. Based on the estimation results we can conclude that scheduling constraints pertaining to the work activity, the time-of-day dependent utility of work, the duration of the pre-work time and the duration of the post-work time (for males) seem to be the most important determinants in deciding how to schedule the work tour. Interestingly, we found support for interactions between activities with respect to duration dependent utility, in the sense that the prework and work activity are complementary and work and after-work appear to be substitutes.

Another important finding is that travel times do not appear significant in the model if time-of-day dependent utility is included in the model. Apparently, travel time primarily seems to be important to the extent that it affects the involvement in work in the period between 8:30 and 10:30 when the marginal utility of work is highest. Including error components results in a significant improvement of the model fit, suggesting that the activity scheduling process that we are modelling is surrounded by much unobserved heterogeneity. The unobserved heterogeneity is found in the valuation of early and late schedule delay and preference for public transport. It should be noted, though, that the heterogeneity may be partly due to the simplified choice settings represented to respondents.

In addition, the valuation of activity involvement varies between socio-demographics segments. Without testing all socio-demographic impacts exhaustively, the results suggest that highly educated individuals value the pre-work time more highly, and that the post-work period is appreciated more by males.

The reported work provides a starting point for further research in various ways. First, more extensive estimation efforts have to be made, including a broader range of socio-demographic and context variables.

Second, as the activity patterns used in this study only entail timing, duration and mode as choice dimensions, more elaborate models need to be estimated which include additional choice dimensions such as activity choice and destination choice. More importantly, data collection and estimation procedures need to be developed that allow for a wider range of activities to be included in the model. This would allow us to estimate more specific utility function that are able to reduce the large amount of unobserved heterogeneity found in the present study. Models based on these extended data and estimation procedures will then constitute a realistic base for the further development of activity-based models.

\section{Acknowledgement}

The use of the data was kindly permitted by the Transport Research Centre of the Dutch Ministry of Transport. 


\section{References}

Arentze, T.A., Timmermans, H.J.P., 2005. ALBATROSS version 2.0. A Learning Based Transportation Oriented Simulation System. EIRASS, Eindhoven.

Ashiru, O., Polak, J.W., Noland, R.B., 2004. The utility of schedules: a theoretical model of departure time choice and activity time allocation with application to individual activity schedules. Transportation Research Record 1894, 84-98.

Becker, G., 1965. A theory of the allocation of time. Economic Journal 75, 493-517.

Ben-Akiva, M., Lerman, S.R., 1985. Discrete Choice Analysis: Theory and Application to Travel Demand. MIT Press, Cambridge, MA.

Bhat, C.R., 1996. A hazard-based duration model of shopping activity with nonparametric baseline specification and nonparametric control for unobserved heterogeneity. Transportation Research B 30, 189-207.

Bhat, C.R., Misra, R., 1999. Discretionary activity time allocation of individuals between in-home and out-of-home and between weekdays and weekends. Transportation 26, 193-209.

Bowman, J.L., Ben-Akiva, M., 1998. Activity-based disaggregate travel demand model system with activity schedules. Transportation Research A 35, 1-28.

Conn, A.R., Gould, N.M., Toint, Ph.L., 2000. Trust-Region Methods. SIAM, Philadelphia, PA.

De Jong, G., Daly, A., Pieters, M., Vellay, C., Bradley, M., Hofman, F., 2003. A model for time of day and mode choice using error components logit. Transportation Research E 39, 245-268.

DeSerpa, A., 1971. A theory of the economics of time. The Economic Journal 81, 828-846.

Ettema, D., 2005. Latent activities: modelling the relationship between travel times and activity participation. Transportation Research Record 1926, 171-180.

Ettema, D.F., Timmermans, H.J.P., 1997. Activity-based Approaches to Travel Analysis. Pergamon Press, Oxford.

Ettema, D.F., Timmermans, H.J.P., 2003. Modeling departure time choice in the context of activity scheduling behavior. Transportation Research Record 1831, 39-46.

Ettema, D.F., Borgers, A.W.J., Timmermans, H.J.P., 1995. A competing risk hazard-model of activity choice, timing, sequencing and duration. Transportation Research Record 1493, 101-109.

Ettema, D., Ashiru, O., Polak, J., 2004. Modeling timing and duration of activities and trips in response to pricing policies. Transportation Research Record. 1894, 1-10.

Evans, A., 1971. On the theory of the valuation and allocation of time. Scottish Journal of Political Economy 2, 1-17.

Hägerstrand, T., 1970. "What about people in regional science?". Regional Science Association Papers 24, 7-21.

Hensher, D.A., Greene, W.H., 2003. The mixed logit model: the state of practice. Transportation 30, $133-176$.

Hess, S., Polak, J.W., Daly, A.J., Hyman, G., 2007. Flexible substitution patterns in models of mode and time-of-day choice: New evidence from the UK and The Netherlands. Transportation 34, 213-238.

Jara-Diaz, S.R., 1998a. Time and income in travel choice: Towards a microeconomic activity based theoretical framework. In: Gärling, T., Laitila, T., Westin, K. (Eds.), Theoretical Foundations of Travel Choice Modelling. Pergamon Press/Elsevier, Oxford/Amsterdam, pp. $1-73$.

Jara-Diaz, S.R., 1998b. A general micro-model of users behavior: The basic issues. In: Ortúzar, J.D., Hensher, D., Jara-Diaz, S.R. (Eds.), Travel Behavior Research: Updating the State of Play. Pergamon Press, Oxford.

Joh, C.-H, Arentze, T.A., Timmermans, H.J.P., 2003. A theory and simulation model of activity-travel rescheduling behavior, Transportation Research A.

Kitamura, R., 1984. A model of daily time allocation to discretionary out-of-home activities and trips. Transportation Research B 18, $255-266$.

Meloni, I., Guala, L., Loddo, A., 2004. Time allocation to discretionary in-home and out-of-home activities and to trips. Transportation 31, 69-96.

Owen, A.B., 1998. Scrambling Sobol' and Niederreiter-Xing points. Journal of Complexity 14, 466-489.

Polak, J., Jones, P., 1994. Travellers' choice of time of travel under road pricing. In: Paper Presented at the 73rd Annual Meeting of the Transportation Research Board, Washington, DC.

Small, K.A., 1982. The scheduling of consumer activities: work trips. The American Economic Review 72, 467-479.

Train, K.E., 2003. Mixed Logit. Discrete Choice Methods with Simulation. Cambridge University Press, Cambridge, pp. 1-334.

Vovsha, P., Bradley, M., 2004. Hybrid discrete choice departure-time and duration model for scheduling travel tours. Transportation Research Record 1894, 46-56.

Winston, G.C., 1982. The Timing of Economic Activities: Time-specific Analysis of Household Activities. Cambridge University Press, Cambridge.

Yamamoto, T., Kitamura, R., 1999. An analysis of time allocation to in-home and out-of-home discretionary activities across working days and non-working days. Transportation 26, 231-250.

Yamamoto, T., Fujii, S., Kitamura, R., Yoshida, H., 2000. An analysis of time allocation, departure time and route choice behavior under congestion pricing. In: Paper Presented at the 79th Annual Meeting of the Transportation Research Board, Washington, DC.

Zhang, J., Timmermans, H., Borgers, A., 2002. A utility-maximizing model of household time use for independent, shared and allocated activities incorporating group decision mechanisms. Transportation Research Record 1807, 1-8. 